\title{
Load-Stress Test of Massive Handovers for LTE Two-Hop Architecture in High Speed Trains
}

\author{
A.Parichehreh $^{(1) *}$, U.Spagnolini ${ }^{(2)}$, P.Marini $^{(1)}$, A.Fontana $^{(1)}$ \\ (1): Prisma Telecom Testing, Milano (Italy) \\ (2): Dip. Elettronica, Informazione e Bioingegneria, Politecnico di Milano (Italy)
}

Abstract

Load-stress test is the experimental performance analysis in extreme traffic and density conditions, routinely required to validate any innovative radio access solution. This paper focuses on load-stress test specifically designed for the two-hop architecture that enables the onboard connectivity in high speed trains (HSTs). The load-stress condition of train-to-infrastructure communication for a massive number of on-board users (UEs) is very challenging, as it needs to account for extreme conditions and complex testing environment. Load-stress method proposed in this paper is for ground-network supporting onboard wireless connectivity in HST, it is validated for commercial evolved NodeBs (eNBs) from Long Term Evolution (LTE) cellular networks (Rel-11).

The in-Lab experimental setup is arranged by virtualizing multiple eNBs serving multiple cells, arranged sequentially along a line to simulate the HST track with a massive number of active onboard UEs. The focus of the experimental load-stress test is the analysis of the impact of Doppler and frequent interruptions caused by the frequent handovers (HOs) of multiple consecutive groups of UEs deployed in HST carriages at the speed of $300 \mathrm{~km} / \mathrm{h}$. The HO interruption time is characterized statistically based on the number of active UEe. The consequent impairments on the experienced QoS for high-throughput and low-latency services such as FTP and VoLTE are verified. This paper validates experimentally the traffic and HO latency improvements (approx. x3) in multi-cell access scheme, when the coverage of every single carriage is augmented by fixed directional antennas to offload the UEs toward far-away eNBs along the train track.

\section{Introduction}

Seamless wireless connectivity in different mobility scenarios is one of the key requirements of 5G networks [1]. Recently, Third Generation Partnership Project (3GPP) has standardized Long Term Evolution (LTE) and its advanced version LTE-A, with a peak data rate up to $100 \mathrm{Mbps}$ in high mobility scenarios ( $\geq 300 \mathrm{~km} / \mathrm{h}$ ), aiming at a trade-off between demanded data rate and quality of service (QOS) for HST onboard passengers [2]. Despite this improvement, the hard handover (HO) nature of LTE system that breaks the connection before connecting to the target evolved NodeB (T-eNB) inevitably causes service interruption, and degrades the users' QoS.

In the HST scenario, QoS of massive onboard UEs (say up to 1000) is highly critical, as affected by different impairments such as frequent HOs, Doppler shift, and limited number of Physical Resource Blocks (PRB). The serving eNB (S-eNB) is

\footnotetext{
*Corresponding author: alip@prismatelecomtesting.com

Prisma Telecom Testing Srl

Via Petrocchi 4, Milano, Italy

Tel: +3902 26113507

Fax:+3902 26113597
} 
highly loaded due to the large number of onboard UEs camped in, and when a cell change happens, the heavy HO signaling load increases both HO-induced latency and link failure rate. This situation worsens at higher speeds due to the Doppler shift and consequently the overall link-quality degradation. Managing this signaling is very challenging as involves a large number of UEs, spatially concentrated in less than $200 \mathrm{~m}$ (i.e., the train length) that moves at the speed up to $500 \mathrm{~km} / \mathrm{h}$ (or equivalently, all onboard UEs cross cell-boundaries in less than $1.5 \mathrm{sec}$ ).

Despite all the research activities on the HST onboard connectivity, due to a lack of a readily available testbed, any innovation in HST connectivity can only be validated either by theoretical analyses and/or system level simulations. Even if these validations can hardly reflect all side-challenges of actual systems [see 3, 4, 5, 6], system level simulations are crucial to highlight the importance of each individual aspect, but experimental measurements and testing with actual eNBs become mandatory to evaluate in-field benefits, possibly in realistic settings before network deployment.

The first preliminary experimental study of LTE mobility management entity (MME) and specifically X2-based HO is provided in [7]. A detailed analysis of the $\mathrm{HO}$ is investigated in [8] and a stochastic model for the HO interruption time (HIT) is extracted via a field-test measurement for different urban scenarios. An experimental study analyzed the HOinduced latency in two slow and high mobility scenarios, up to $100 \mathrm{~km} / \mathrm{h}$ [9]. However, in publicly available literature the network is loaded at most by 8 users per cell, and this load value is estimated as the experimental setup is not under complete control. This setup is not sufficient to measure the performance of the LTE network in extreme load-conditions, especially in HST with up to 1000 UEs at the speed of $300 \mathrm{~km} / \mathrm{h}$ or more. In addition, these experiments lack control on the other active UEs' traffic and mobility profile.

Instead of conducting in-field measurements, we design holistic in-Lab experiment to evaluate the performance of MME of the LTE network with off-the-shelf eNB, while providing high throughput and/or low latency Internet services for massive number of HST onboard UEs. In these experiments, mobile UEs travel at the speed of $300 \mathrm{~km} / \mathrm{h}$ and the eNBs are frequently pushed to their load-limits in a fully-controlled environment. To the best of our knowledge, this is the first inLab experimental study in open literature focusing on the performance analysis of the actual LTE network along the HST railway, aiming at extracting the statistical characteristics of the frequent HOs and its impairment on the throughput, link failure, and call drop rate.

A load-stress test equipment for actual eNBs based on software defined radio is adapted to account for this critical mobility condition. The experimental setting is extended to validate different onboard relaying architectures for HST as specified by 3GPP [10], with conventional omnidirectional antennas and multi-cell access scheme provided by directional antennas along the railway [4].

\section{Preliminaries}

In this introductory section, we briefly detail the HST train-to-infrastructure connectivity, the HO procedure and the multi-cell access scheme proposed to cope with massive HOs.

\subsection{Two-hop architecture for High Speed Train}

Recently, 3GPP has standardized a two-hop architecture (see Fig.1) for HST onboard connectivity via relay nodes. Onboard relay nodes can be classified either into Layer-1 (L1) relay or mobile relay node (MRN) [12], based on their functionalities. 
L1-relay is a bidirectional amplify-forward relay that bridges the onboard signals with the eNBs after some power equalization. L1-relay can be considered as a low-cost solution to enable onboard connectivity, routinely deployed on HST for $2 \mathrm{G}$ and $3 \mathrm{G}$ networks, and for LTE Rel-8. In addition to the common disadvantages of amplify-forward relay, it leaves the mobility management of massive UEs to be carried out individually on each UE, and this is a remarkable drawback, as detailed later. MRNs from LTE Rel-12 are basically small-cells acting as autonomous (and mobile) eNBs located in each carriage of HST. The onboard eNB acts as a compound UE that is, in turn, connected to the Donor-eNB via Un interface. Compared to L1-relay, MRN is more complex and expensive. However, it provides one connection per carriage with group-HO feature that performs one single $\mathrm{HO}$ for all the connected UEs, resulting in a lower $\mathrm{HO}$ impairment and higher throughput per UE. The drawback of MRN is that all the UEs will lose their connection in case of any link failure.

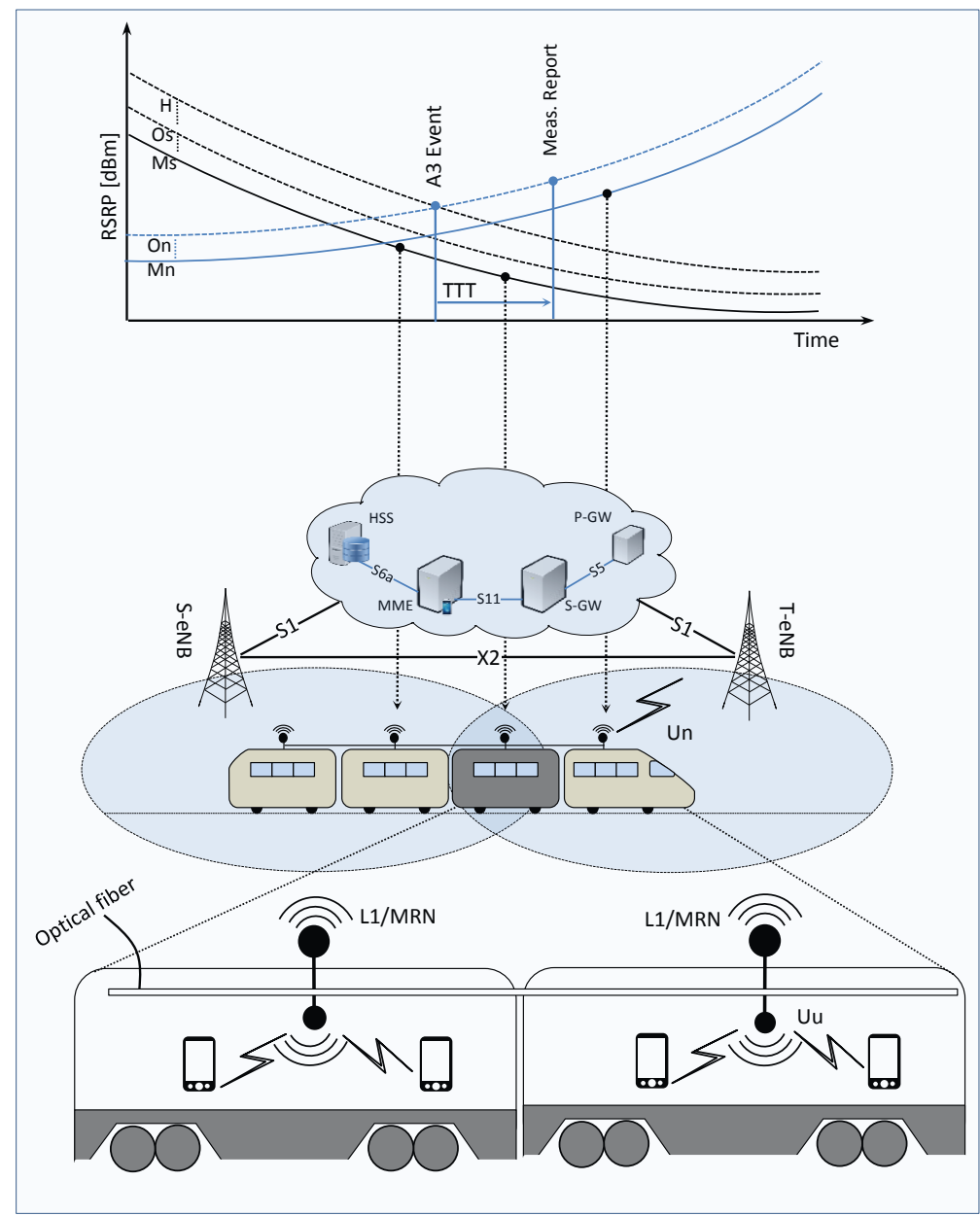

Fig.1: Two-hop architecture of HST on-board LTE network. L1/MRN [10] on the roof of the HST. Inter-carriages communication through optical fiber, if present/necessary. A3-event for $\mathrm{HO}$ and related parameters (on top).

\subsection{LTE handover on HST}

Generally, HO procedure includes three phases: HO preparation phase (i.e., measurement report by UE and HO decision by eNB), $\mathrm{HO}$ execution phase (i.e., Random Access Channel (RACH) procedure and synchronization to the TeNB, and receiving uplink grant), and HO completion phase (i.e., path switching and bearer modification).

According to LTE-A Radio Resource Control (RRC) specification [13], UEs measure the Reference-Symbol-ReceivedPower (RSRP) of the neighboring cells based on the configuration in RRC_Connection_Reconfiguration message 
received from the S-eNB. This message includes i) a list of neighbor eNBs to be measured and ii) reporting mechanism (i.e., periodical or event-based measurement). In periodic measurement UE measures the RSRP value periodically, while in event-based measurement, UE sends the measurement report once a certain event is triggered. Different types of events (labelled A1 to A5) can be configured in RRC_Connection_Reconfiguration message to trigger a measurement report for different purposes. Among them, A2 and A3 events are commonly used for HO procedure. A2event is used by UE for inter-frequency HOs, to inform the eNB that the RSRP value of S-eNB is below a predefined threshold, and receive the required measurement gaps necessary for inter-frequency HOs. A3-event is the same for IntraFrequency HOs, and this is the setup used here for load-stress test (Sect.3).

As shown in Fig.1, A3-event happens when measured RSRP for neighbor cells (Mn) is larger than for the serving cell (Ms) up to some offset values (Os and On, for S-eNB and potential T-eNBs, respectively) and hysteresis value (H). In other words, $\mathrm{Mn}+\mathrm{On}>\mathrm{Ms}+\mathrm{Os}+\mathrm{H}$. S-eNB makes the $\mathrm{HO}$ decision according to the received measurement report, and sends information of the T-eNB to the UE through RRC_Connection_Reconfiguration message to start HO execution phase over RACH. The HIT is the interval from the time instance that the UE receives an RRC_Connection_ Reconfiguration message to the time that it sends RRC_Connection_Reconfiguration_Complete message to the T-eNB, after receiving uplink grant. Finally, $\mathrm{HO}$ completion phase according to the type of HO (e.g., intraeNB, X2 or S1) will be executed.

We remark that, considering the specified two-hop architecture of HST connectivity, all the UEs inside each carriage, experience the same Ms and Mn values. This is due to the fact that all the devices are served by the same wireless link provided by onboard relay (L1/MRN). Therefore, A3-event will be triggered for all the UEs simultaneously. This potentially causes a bottleneck in the HO execution phase over RACH channel.

\subsection{Directional antennas for Multi-Cell access scheme}

The purpose of multi-cell access (MA) scheme of the train is to deploy multiple directional antennas on each carriage that diversify the wireless connectivity over multiple eNBs [4]. Fig.2-b) shows the MA scheme that is compatible with either L1 or MRN onboard relaying. Onboard UEs can be connected to different antennas that are either omnidirectional or directional pointing forward and backward to the remote cells. These directional antennas have fixed beams that point towards lightly loaded far eNBs, compared to the omnidirectional antenna for the local eNB the carriage is camped in. A dispatcher balances the onboard UEs over the three cells (and the associated eNBs), depending on UE traffic intensity and ground eNB cell load. The MA scheme reduces the HO signaling of the local S-eNB, while distributing the carriage load among multiple eNBs and achieving higher capacity. Namely, the HO decision can be manipulated by the spatial signal propagation of the directional antennas without any necessary coordination from the LTE eNB.

Compared to the current two-hop architecture [10], the directional antennas placed on each carriage that point toward less loaded neighbor cells, manipulate the cell shape according to the directivity pattern (Fig.2-b). Directional antennas do not need to be adaptive as the track is mostly straight to avoid lateral passengers' accelerations at 300$500 \mathrm{~km} / \mathrm{h}$, and eNBs are customary deployed off-track, close to the track for maximum efficiency distance $\mathrm{h}=50 \mathrm{~m}$ [10]. This simple method provides an augmented number of resources (i.e., PRB and PRACH channels) for massive onboard UEs that, in turn, increases data rate and shortens the HIT for on-board UEs. 


\section{Load-stress test for HST: case study and experiments}

Rather than having a collection of UEs onboard the HST, the in-Lab system is based on the adaptation of the UeSIM, a load-stress testing equipment for LTE radio interface and core network [14]. UeSIM is a commercial load and stress testing system able to load an eNB on the RF interface, simulating thousands of UEs each with a different channel and traffic model toward the same eNB (or two eNBs when in the HO region). The test evaluates the individual/aggregate QoS of massive onboard UEs in HST scenario, using commercially available eNBs with individual UEs supporting high throughput and/or low latency services such as File Transfer Protocol (FTP) and Voice over LTE (VoLTE). The UeSIM system in Fig.2, is capable to load up to 8 sectors supporting all frequency bands including FDD and TDD frame types. Besides customized Wireshark packet analyzer that is used to capture packets at the Uu radio interface, UeSIM provides counters at each protocol layer, aggregated at UE, group of UEs or eNB level to measure the network performance while simulating the mobility of the HST. The main benefit from using in-lab testing instead of in-field experiment is that the number of UEs and their parameters can be individually configured from the design of the deployment scenarios to the UE behavior (from physical layer up to the application layer parameters, radio bearer type, and mobility/traffic profiles).

The network configuration for the HST load-stress test is in Table 1. The LTE air-link operates at $7^{\text {th }}$ frequency band $(2.6 \mathrm{GHz})$ with FDD frame structure. The bandwidth is $10 \mathrm{MHz}$ and the simulated UEs are from category 4 , supporting $2 \times 2$ MIMO and up to $150 \mathrm{Mbps}$ in downlink (DL) when using 64-QAM modulation and coding scheme. There are two HST scenarios of N UEs with homogeneous traffic: i) each UE downloads a 1GB file from an external FTP server through LTE eNB over default bearer; ii) each UE is configured to establish a Voice over LTE call (VoLTE) over dedicated bearer with guaranteed bit rate throughput. The UeSIM emulates the behavior of N UEs with individual LTE protocol stack from Non Access Stratum (NAS) protocol (3GPP-TS 24.301) to the physical layer baseband processing (3GPP-TS 36.104) according to the LTE specification (Rel-11), running over software defined radio module controlled by a multicore Linux machine. Propagation channel (e.g., path-loss, fading, multipath, Doppler shift and noise) is simulated by controlling the impairments individually for each UE at the level of baseband IQ stream, as shown in Fig.2-c. The propagation over the air-link is replaced by a coaxial cable (to avoid illegal frequency radiation) toward a 2×2 MIMO eNB. In the conducted experiments, we consider a train with $M=4$ carriages in a rural area and $N$ UEs $(N=32,40,60,72,88,120)$ moving at $v=300 \mathrm{~km} / \mathrm{h}$ over a $200 \mathrm{Km}$-track (total run-time is $40 \mathrm{~min}$ ) of $\mathrm{K}$ sequential eNBs (here $\mathrm{K}=200$ ) with cell size $\mathrm{D}=1 \mathrm{Km}$ (according to the open space scenario specified in [11]). All the $\mathrm{K}$ eNBs are placed in the middle with omnidirectional antennas, as shown in Fig.2-a. Given these specified parameters, the train crosses cell-boundaries every 12s. Each carriage has $N / M=\{8,10,15,18,22,30\}$ UEs on the same carrier frequency. Inter-carriage HO delay is 300ms (i.e., time interval for two consecutive carriages for crossing the cells, assuming a carriage-length of $25 \mathrm{~m}$ ).

To carry out a holistic experimental study on the impact of massive onboard UEs on the HIT and TCP DL-throughput of the LTE network, we extract a statistical model of HIT, and verify the impact of the HIT on the FTP DL-throughput for the ensemble of all the N UEs by measuring the instantaneous Physical Downlink Shared Channel (PDSCH) throughput dedicated to the FTP services. We first disable the Doppler shift to evaluate the HIT and the impact of HO on FTP throughput, then the effect of Doppler shift on the link and service failure is individually investigated for VoLTE service. 


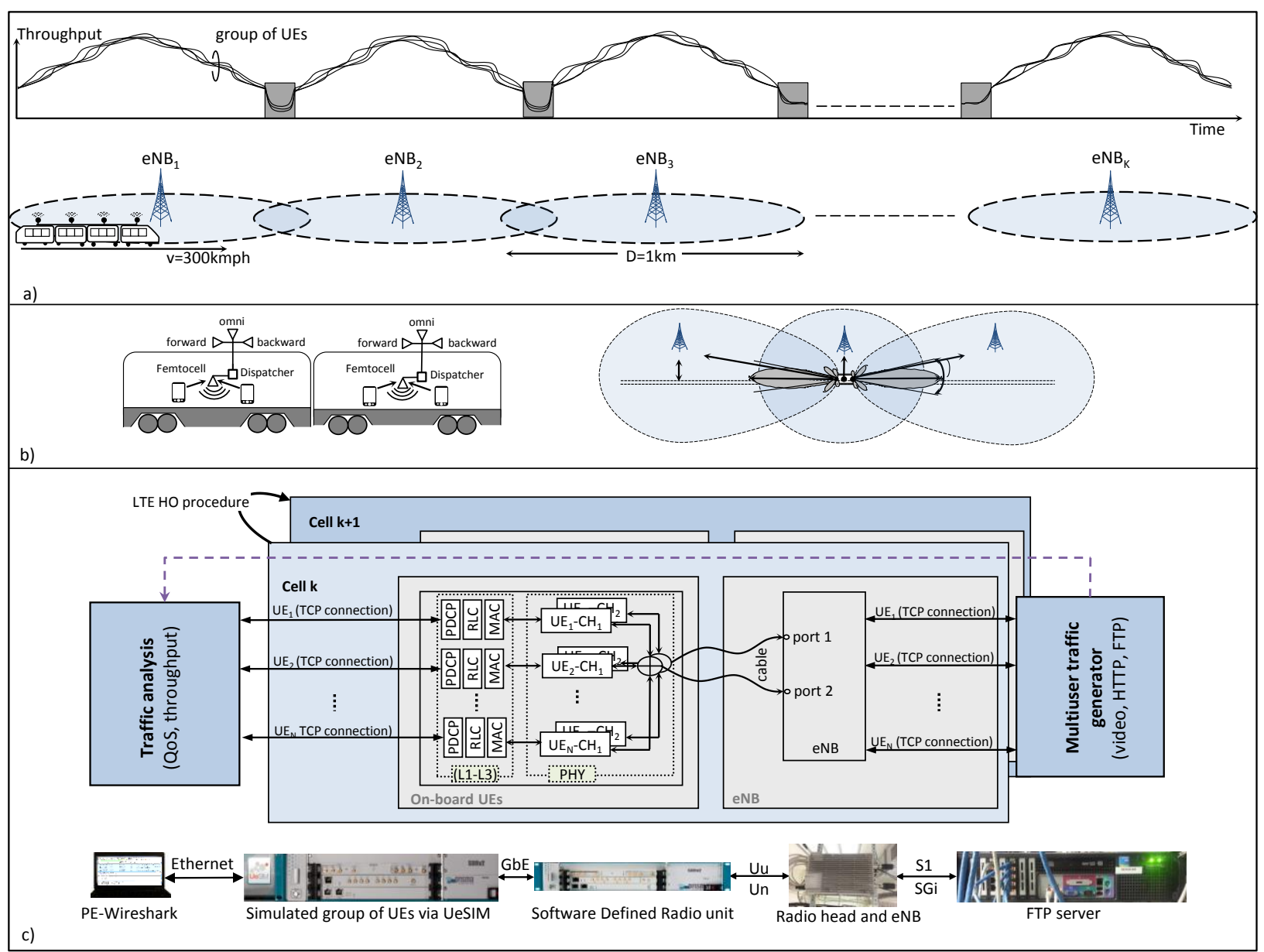

Fig.2. in-Lab experimental setup overview: (a) HST with $\mathrm{K}=200$ eNBs, cell-size $\mathrm{D}=1 \mathrm{Km}, \mathrm{v}=300 \mathrm{~km} / \mathrm{h}$, full-penetration loss, and instantaneous throughput of PDSCH for each UE (on the top), and HO (gray shaded area); (b) Two-hop architecture with augmented onboard relay node (L-1 or MRN) with directional antennas to enable the Multi-cell Access (MA) scheme for two-hop architecture (top view, single carriage). (c) UeSIM architecture for $N$ UEs performing intrafrequency $\mathrm{HO}$ from S-eNB to T-eNB (cell k toward cell k+1) with traffic generation and monitoring, and radio-frequency channel emulator [14].

TABLE. 1. A SUMMARY OF TEST CONFIGURATION

\begin{tabular}{|c|c|c|c|c|}
\hline \multicolumn{5}{|c|}{ Network Configuration } \\
\hline \multirow{2}{*}{ eNB } & \multirow{2}{*}{$\begin{array}{l}\text { LTE }(\text { Rel-11) } \\
\text { band } 7\end{array}$} & downlink & uplink & bandwidth \\
\hline & & $2620 \mathrm{MHz}$ & $2500 \mathrm{MHz}$ & $10 \mathrm{MHz}$ \\
\hline Cell size & \multicolumn{4}{|c|}{$1 \mathrm{~km}$} \\
\hline Off-track distance & \multicolumn{4}{|c|}{$50 \mathrm{~m}[11]$} \\
\hline UE category & \multicolumn{4}{|c|}{ Cat 4, 2×2 MIMO, 150Mbps (with 64QAM MCS) } \\
\hline Mobility scenario & \multicolumn{4}{|c|}{$300 \mathrm{Km} / \mathrm{h}$} \\
\hline Number of UEs & \multicolumn{4}{|c|}{$8,10,15,18,22,30$} per carriage \\
\hline Internet service type & \multicolumn{4}{|c|}{ FTP, VoLTE } \\
\hline \multicolumn{5}{|c|}{ HO Configuration } \\
\hline Hysteresis & \multicolumn{4}{|c|}{ OdB } \\
\hline A3-offset & \multicolumn{4}{|c|}{$2 \mathrm{~dB}$} \\
\hline Time-To-Triger & \multicolumn{4}{|c|}{$40 \mathrm{~ms}$} \\
\hline \multicolumn{5}{|c|}{ RACH Configuration } \\
\hline PRACH configuration index & \multicolumn{4}{|c|}{3} \\
\hline Total number of dedicated preamble & \multicolumn{4}{|c|}{56} \\
\hline Maximum preamble transmission & \multicolumn{4}{|c|}{10} \\
\hline RA response window size & \multicolumn{4}{|c|}{$8 \mathrm{~ms}$} \\
\hline
\end{tabular}


Each load-test emulates the HST over the $200 \mathrm{~km}$ track by extracting the individual and aggregated statistics of UEs with sampling granularity approx. 1s. For every HST run, each UE performs 200 HOs and LTE network fulfils Nx200 HO requests. Fig.2-a exemplifies the instantaneous PDSCH throughput of a subset of UEs with respect to the HST position along the track. The communication link between onboard UEs and eNBs has been designed to emulate the two-hop architecture when employing the onboard relay on top of each carriage, either with L1 relay or mobile relay node (MRN). The set of eNBs to evaluate the massive HOs are configured to handle multi-sectors and the in-Lab setup is configured to have the train virtually move in circle, flipping from one eNB to another.

\section{Experimental results}

\subsection{HO latency model and distribution}

The analysis of RRC messages reveals that A3-event based measurement of RSRP is configured for inter- and intrafrequency HOs, according to the predefined HO parameters: hysteresis and time-to-trigger. When HST moves across cells, the S-eNB receives measurement report for the RSRP from UE, to fulfil the HO decision procedure. Then S-eNB sends the required $\mathrm{RACH}$ resource (including a new C-RNTI, dedicated preamble index, $\mathrm{RACH}$ response windows size, etc.) to allow the UE to switch from the S-eNB to a T-eNB. Note that dedicated RACH preamble is optional and without a dedicated RACH preamble from eNB, each UE selects any random preamble to transmit over contention-based RACH channel. Here, eNBs are configured to send the dedicated preamble following the contention-free RACH procedure.

Even though there is a minimum deterministic delay, $\mathrm{HO}$ interruption time $T_{\text {off }}$ can be modelled as a random variable depending on a complex set of parameters such as the speed of HST, cell size and cell load [4]. For the HST travelling at $v=300 \mathrm{~km} / \mathrm{h}$, each UE experiences a random but periodic HIT at cell-change (every $\mathrm{T}=12 \mathrm{~s}$ ). Hence, for each carriage the off-service interval $T_{\text {off }}$ occurs periodically and varies randomly. This causes a cyclostationary service model for each UE that experiences the HIT every T second. Moreover, for HST at the speed of $300 \mathrm{~km} / \mathrm{h}$ with carriage length $\mathrm{d}=25 \mathrm{~m}$, the HO slides backward over the consecutive carriages every 300ms. The HO-induced latency impairs the onboard UEs' QoS gradually, and worsens over the last carriages. Thus, the QoS for onboard users depends on their carriage-position, the speed of the train and the degree of overlapping of HOs among the consecutive carriages.

The HO properties for massive UEs connected via L1-relay node (currently deployed on the HSTs) are characterized based on the statistical analysis of the HITs vs the number of UEs for nx4 scenarios ( $n$ UE on each carriage and 4 carriages) in Fig.3. HIT $T_{o f f}$ can be modeled as a random variable with Nakagami distribution in accordance with the exact number of the onboard UEs regardless of some minimum latency due to inherent signaling delay [13]. Distribution in Fig.3-a) shows that the QoS impairment is dominated by the tails of the probability density function of $T_{\text {off }}$, and spread parameter $\Omega$ of Nakagami distribution captures this impairment effectively. Note that $\Omega$ varies depending on the number of UEs, T-eNB load and the speed of the HST, spread $\sqrt{\Omega}$ linearly increases with $\mathrm{N}$ as highlighted in Fig.3-b. This is due to the limited number of PRACH resources dedicated to each eNBs for HO signaling, and in test bed we allocated 56 preambles in each eNB reserved for the RACH procedure with 10ms RACH period, wherein only two preambles will be detected and responded by eNB in each RACH channel. Therefore, the successful HO for $70 \%$ of the 120 UEs (in $30 \times 4$ scenario) over contention-free RACH channel takes at least $600 \mathrm{~ms}$ (without preamble miss detection). Compared to urban scenarios (low mobility) where the HOI follows a Normal distribution [8], in-Lab experiments for HST (high mobility) 
scenario with frequent and simultaneous HOs are better described by the Nakagami distribution that accounts for heavy tails of the HITs. Similarly to low-mobility [8], the mean value and standard deviation of the HIT in Fig.3-b are linealy increasing with cell load, with higher rate compared to [8]. Results for other scenarios (e.g., 10x4, 18x4) confirms the results so far.

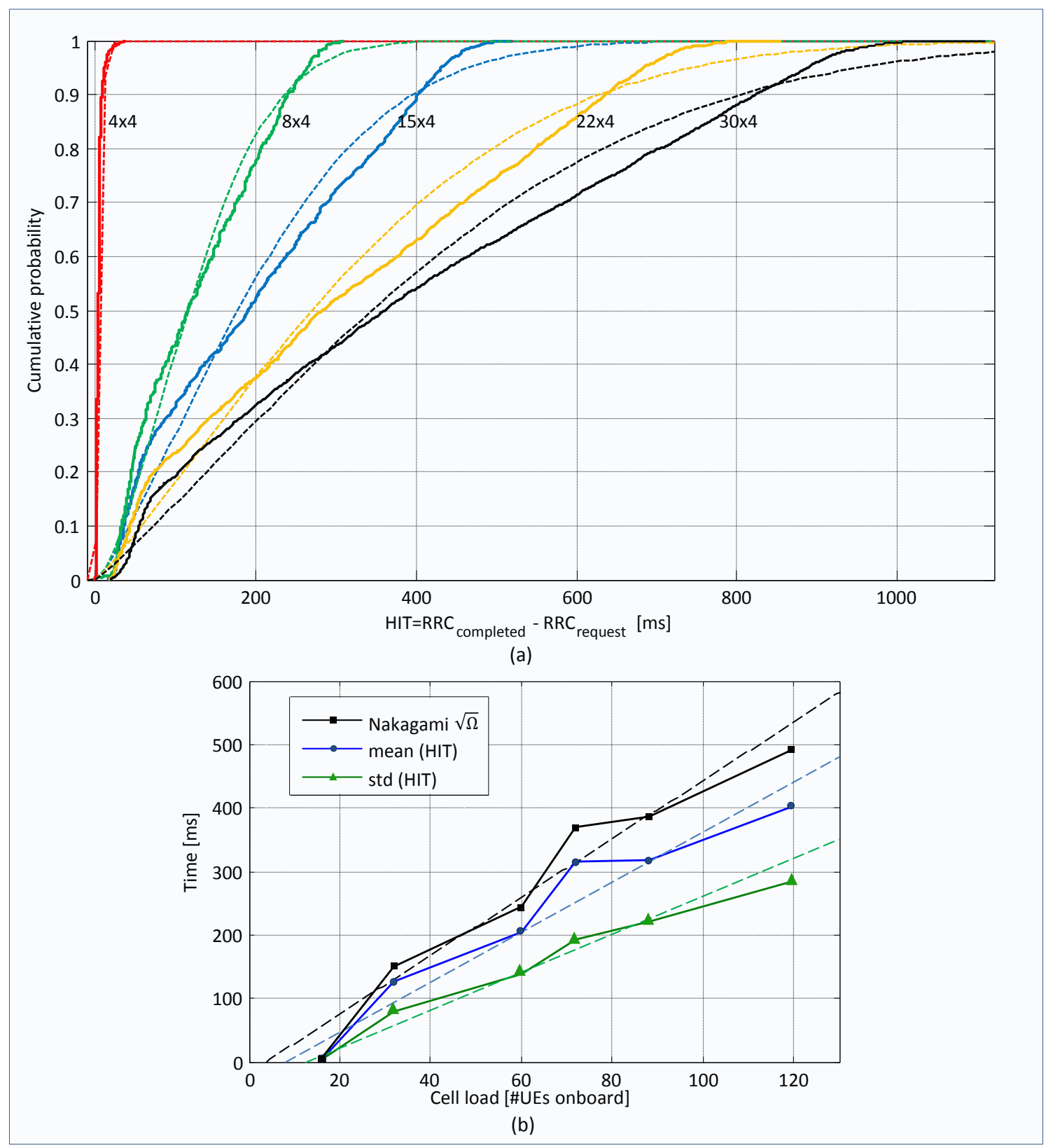

Fig.3. HO interruption time analysis versus the number of UEs for L1 relay architecture: (a) Empirical CDF of HIT (solid lines) and Nakagami distribution fitting (dashed lines); (b) Nakagami spread control parameter $\sqrt{\Omega}$, mean value and standard deviation of the HITs versus the cell load, linear fitting vs UEs (dashed line). 

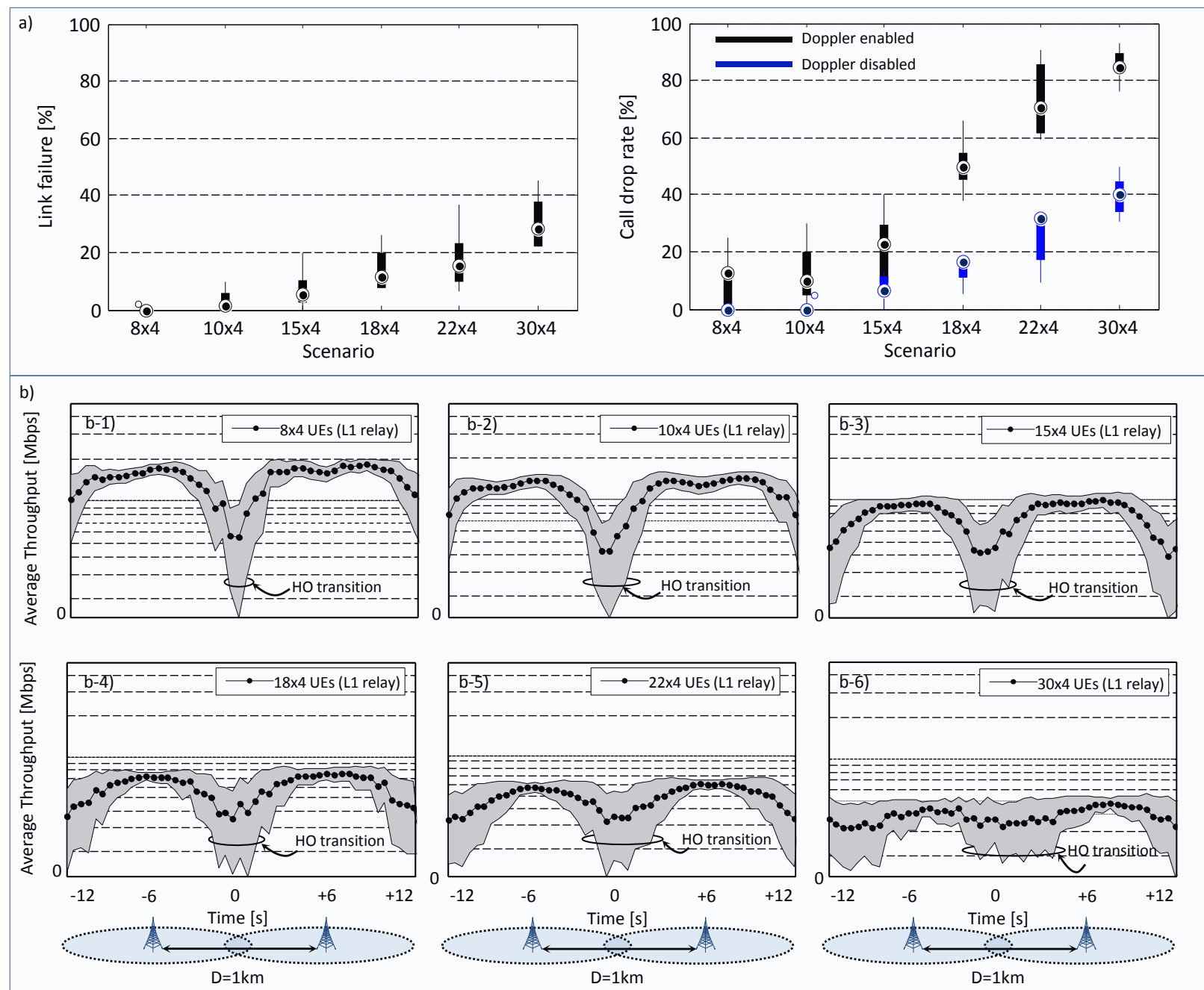

Fig. 4. (a) Effect of massive handovers on link failure and Voice over LTE (VoLTE) call drop rate; (b) Effect of the massive onboard UEs on the PDSCH throughput ( \pm standard deviations in shaded-gray area); HST with $\mathrm{M}=4$ carriages and $\{8,10$, $15,18,22,30\}$ UEs per carriage, at the speed v=300km $/ \mathrm{h}$. UEs are connected to the eNBs through L1-relay.

\subsection{HO impairment on Link Failure and VoLTE call drop rate}

The effect of the HOs from massive number of onboard UEs on the link failure rate (when Doppler shift is enabled) is shown in Fig. 4.a. Link failure is the result of the HO failure (owing to preamble miss detection). Considering the small offtrack distance, at the speed of $300 \mathrm{~km} / \mathrm{h}$, Doppler shift varies between $[-722 \mathrm{~Hz},+722 \mathrm{~Hz}]$ in downlink, and $[-1444 \mathrm{~Hz}$, $+1444 \mathrm{~Hz}$ ] in uplink direction (i.e., each UE estimates the channel comprehensive of Doppler shift in downlink, and replies with that frequency shifted synchronization in uplink that sums to the Doppler). In order to have a tractable and reproducible measurement and analysis, here we assume that the train is affected by a fixed Doppler, although in reality the speed of train in not fixed over the track and Doppler transition when crossing the eNB imposes severe impairments on the link quality. Due to the Doppler shift and transition effects, we experienced that T-eNB fails to detect the dedicated preambles and UEs fail to accomplish the RRC reestablishment procedure. Consequently, links fail and the link failure rate increases significantly in high loading scenarios. This is shown in Fig.4-a, up to $40 \%$ of the mobile users experience link failure in the scenario with $30 \times 4$ mobile UEs. 
In order to evaluate the effect of link failure on QoS, all UEs use VoLTE service with narrowband G.711 audio codec over RTP protocol and dedicated bearers with QoS Class Identifier, QCl=1 (45kb/s guaranteed bit rate in UL and DL directions). Open Source Kamailio is the session initiation server (SIP). Fig.4.a illustrates the call-drop without Doppler (only HO-interruptions) and with Doppler enabled vs the increasing the number of mobile users, VoLTE call drop rate dramatically increases. When 120 UEs are onboard the HST the call drop rate is $90 \%$ and service is useless. Again, this impairment is not only due to the link failure but also long latency induced by HO (note that packet delay budget for $\mathrm{QCl}=1$ is $100 \mathrm{~ms}$, while the $\mathrm{HO}$ latency lasts up to $1000 \mathrm{~ms}$ ). At cell boundary there is a congestion on the RACH channel (beside path loss attenuation at cell boundaries), and SIP server assumes that channel quality is not acceptable and drops the call with standard cause-code $=6$ (i.e., unacceptable channel according to SIP specification). This accurately reflects the nature of the internal failure over radio interface at cell boundaries. The comparison of call drop rate with Doppler disabled shows quantitatively the compound effects of each term into the QoS.

\subsection{HO impairment on the FTP DL-throughput}

The effect of the HO over the throughput of the massive onboard UEs is shown in Fig.4-b. Due to the cyclostationary behavior of the throughput, collected data are illustrated around the HO occurrence point (used for time reference: $t=0$ ). Fig.4-b highlights that at the cell-edges the average throughput drops due to the simultaneous HOs, and UEs experience the maximum throughput inside the cell that scales by $1 / \mathrm{N}$ (70Mbps/N per UE) and it decreases to $300 \mathrm{kbps}$ when increasing the number of active UEs up to $30 \times 4$. The $8 \mathrm{~Kb} / \mathrm{s}$ traffic of system information block transmission on the PDSCH is not accounted into the per-user throughput.

When cells are heavy-loaded, as for 30×4 (120 UEs over 4 carriages), the fluctuations of throughput (shaded-gray area) increases due to the multiple HO transitions that leave the UEs in outage for long time intervals (up to $1 \mathrm{~s}$ for $30 \times 4$, see Fig.3). More precisely, the limited the number of preamble for $\mathrm{HO}$ signaling in one side, and eNB limited capability in answering the $\mathrm{HO}$ requests on the other side, avoid T-eNB to response all the HO requests in a timely manner. In fact, the S-eNB buffers the incoming measurement reports and responses only two of them in every RACH period. Therefore, additional delay overhead on the RACH channel is inevitable (especially for the UEs located in the tail carriages of the train) that accordingly effects on the congestion control procedure on transport layer and shortens the FTP window size, owing to unacceptable link quality at cell boundaries.

Note that this part of experimental result is purposely without Doppler shift to isolate the impact of the frequent HOs on the QoS of onboard users versus the number of UEs. Since Doppler degrades the overall performances considerably (with higher preamble miss detection probability and lower throughput), the results here are the reference bound for the UEs density and the specific eNB manufacturer. Note that including Doppler shift in this experiment could cause link failure which augments the instability in the experiment and prevents the HO analysis.

\section{Load-test for Multi-cell Access scheme:}

In L1-relay when increasing the number of UEs, the throughput per each UE decreases quite dramatically due to the mobility managements for massive UEs treating independently one from another. MRN copes with this drawback by leaving one $\mathrm{HO}$ per cell, thus gaining all the benefits in term of throughput as shown in Fig.5-a) for 30x4 UEs load. However, the MA scheme can reduce the massive UEs impairment providing higher throughput and HO signaling performance by diversifying the traffic load over three eNBs at the same time, possibly with no-overlapping HO. 
The load-test results for massive UEs can be extrapolated to infer the performance of Multi-cell Access (MA) scheme with two additional onboard relays connected to two different eNBs located ahead and behind the S-eNB that train is located. Experimental results on the same setup (only 30x4 is shown here) are in Fig.5-a, it proves that the MA scheme increases the throughput per UE up to approximately three times more compared to the conventional relay with one omnidirectional antenna per carriage. The use of 3 MRN per carriage equipped with omni/directional antennas (MRN+MA) outperforms the other schemes due to the group-HO feature that reduces the HO time for the onboard UEs. Furthermore, the L1-relay with MA scheme is a simple and effective solution for the massive UEs provided that mobility management is redesigned to account for the independent UEs to camp over far-ahead cells. As a drawback, the L1+MA suffers from the cumbersome HO signaling that causes an increasing HO failure rate (around 2-6\% for 88-120 onboard UEs for the L1-relay), while the HO failure was negligible for the MRN.

In L1-relay with omnidirectional antenna the eNB should manage $120 \mathrm{HOs}$ almost simultaneously, and this extends the $\mathrm{HO}$ time up to 1s (Fig.5-b) as a consequence of to the limited number of PRACH channels dedicated to HO procedure in each eNB. However, in the scenario with the L1-relay node with augmented coverage with MA scheme, it can be observed that the $\mathrm{HO}$ time lasts no more than $300 \mathrm{~ms}$. In fact, MA scheme diversifies the HO signaling over multiple cells. In the HST scenario equipped with MRN, the HIT is less than $40 \mathrm{~ms}$ and this meets the IMT-Advanced HO requirements, namely $\leq 60 \mathrm{~ms}$. In MRN the multi-cell access scheme has no practical impact on HO (same as HO time of a single UE) but rather on throughput as shown in Fig.5-a.

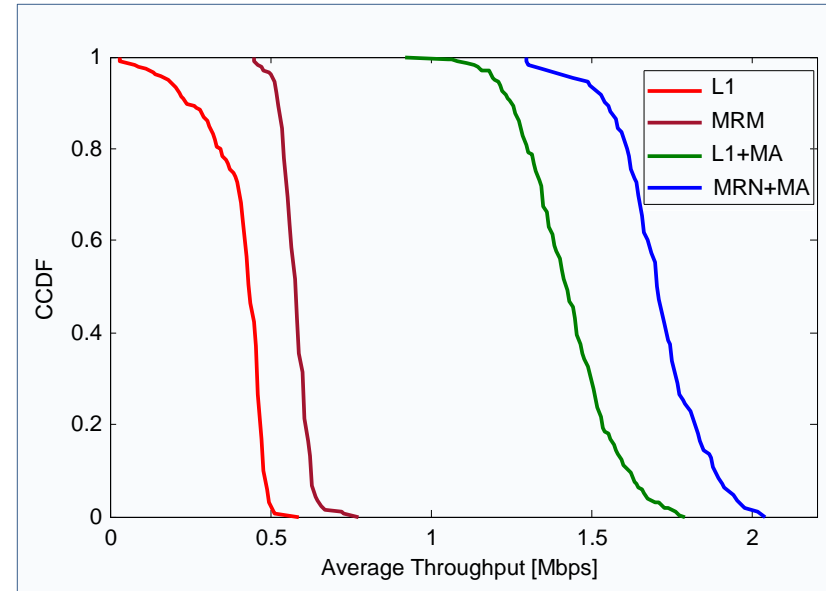

(a)

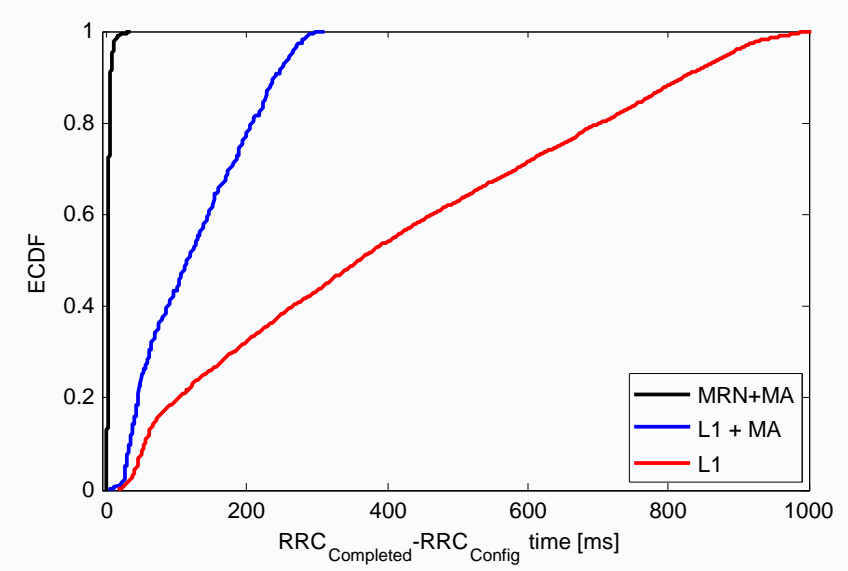

(b)

Fig.5: Cumulative Distribution Function (CDF) of FTP DL-throughput per UE (a), and HO time (b) for different relay technologies and multi-cell access (MA) method for the heavy-load case of 30 UEs per carriage (case $30 \times 4$ ).

\section{Conclusion}

In this paper, we introduced a novel ad-hoc load-stress testing method to analyze the performance of the LTE eNBs in high mobility scenarios, when onboard connectivity is established over two-hop train-to-infrastructure architecture. We extracted the statistical characteristics of the X2-based HO interruption time and its impairments on the throughput, link failure and VoLTE call drop rate. The HO interruption time for each onboard UEs is modeled by a Nakagami distribution, in which the spread control parameter depends the cell load and number of active UEs. The in-Lab experiment at $300 \mathrm{~km} / \mathrm{h}$ (but extension of set-up to higher speed is conceptually straightforward) highlighted quantitatively how the quality of service at the cell-edge reduces due to the HO-induced latency. The augmentation of onboard relay nodes (L1/MRN) with 
fixed directional antennas can remarkably enhance by x3 the QoS of massive UEs by offloading the traffic over lightly loaded cells, with faster HO responses and larger throughput.

The load-stress test highlighted some areas of improvements in HST communication: i) adaptation of network mobility management by reducing the RA response period and/or increasing the RACH processes in eNB (to manage more RACH requests at the same time), ii) offloading the traffic among the carriages during the HO interruption time, and iii) optimization of MA scheme via beam-width coordination of directional antennas.

\section{References:}

[1] 5 G Forum white paper, “5G Vision, Requirements, and Enabling Technologies,” (v.2.0), 2016.

[2] A. Ghosh, R. Ratasuk, B. Mondal, N. Mangalvedhe, T. Thomas, "LTE-advanced: Next-Generation Wireless Broadband Technology," IEEE Wireless Commun., 2010, vol. 17, no. 3, pp. 10-22.

[3] A. Sniady, J. Soler, M. Kassab, M. Berbineau, “Ensuring Long-Term Data Integrity: ETCS Data Integrity Requirements Can Be Fulfilled Even under Unfavorable Conditions with the Proper LTE Mechanisms," IEEE Veh. Technol. Mag., vol. 11, no. 2, 2016, pp. 60-70.

[4] A. Parichehreh, S. Savazzi, L. Goratti, U. Sspagnolini, “Seamless LTE Connectivity in High-Speed Trains," Wireless Commun. Mobile. Comput., 2015, vol. 16, no. 12, pp. 1478-1494.

[5] T. Han, N. Ansari, "Radio over Fiber as an Antenna Extender for High-Speed Train Communications," IEEE Wireless Commun., 2015, vol. 22, no. 1, pp. 130-137.

[6] D. Xenakis, N. Passas, L. Merakos, C. Verikoukis, "Mobility Management for Femtocells in LTE-Advanced: Key Aspects and Survey of Handover Decision Algorithms," IEEE Commun. Surveys Tuts., 2014, vol. 16, no. 1, pp. 64-91.

[7] A. Elnashar, M. A. El-Saidny, "Looking at LTE in Practice: A Performance Analysis of the LTE System Based on Field Test Results," IEEE Veh. Technol. Mag., 2013, vol. 8, no. 3, pp. 81-92.

[8] D. Han, S. Shin, H. Cho, J. Chung, D. Ok, I. Hwang, "Measurement and Stochastic Modeling of Handover Delay and Interruption Time of Smartphone Real-Time Applications on LTE Networks," IEEE Commun. Mag., 2015, vol. 53, no. 3, pp. $173-181$.

[9] L. C. Gimenez, M. C. Cascino, M. Stefan, K. Pedersen, A. F. Cattoni, “Mobility Performance in Slow- and High-Speed LTE Real Scenarios," IEEE VTC Spring 83rd, Nanjing, China, 2016.

[10] 3GPP TS 36.836: "Technical Specification Group Radio Access Network; Evolved Universal Terrestrial Radio Access (E-UTRA); Study on Mobile Relay (Release 12)," http://www.3gpp.org/, [Accessed on Dec. 20, 2016].

[11] 3GPP TS 36.104: "Technical Specification Group Radio Access Network; Evolved Universal Terrestrial Radio Access (E-UTRA); Base Station (BS) radio transmission and reception (Release 13)," http://www.3gpp.org/, [Accessed on Dec. 20, 2016].

[12] Y. Sui, J. Vihriälä, A. Papadogiannis, M. Sternad, W. Yang, T. Svensson, “Moving Cells: a Promising Solution to Boost Performance for Vehicular Users," IEEE Commun. Mag., 2013, vol. 51, no. 6, pp. 62-68.

[13] 3GPP TS 36.331: “LTE; Evolved Universal Terrestrial Radio Access (E-UTRA); Radio Resource Control (RRC); Protocol specification, (Release 13)," http://www.3gpp.org/, [Accessed on Dec. 20, 2016].

[14] Prisma white Paper, "UeSIM: Multi-UE Simulation over Radio Interface with a Single Device," http://www.prismatelecomtesting. com/, [Accessed on Dec. 20, 2016]. 